\title{
Health and Sustainable Development
}

\author{
Rahman MR
}

The spectrum of health, environment and development hazards has changed considerably over the millennia of human existence. In the past 50 years in particular, the world has seen considerable health gains. For example, childhood mortality and morbidity have been greatly reduced by better control and prevention of infectious diseases. People are living much longer. Between the $1950 \mathrm{~s}$ and the 1990s, average life expectancy increased from 46 to 65 years, and the gap in life expectancy between rich and poor countries narrowed considerably, from 25 years in 1955 to 13.3 years in $1995^{1}$.

There have been major advances in science and technology and health and medicine, infrastructure has expanded, literacy has increased, education has improved and incomes and opportunities have increased, especially for women. Yet, despite all this, in many instances the health gaps between and within countries are widening. Not all regions of the world have shared equally in improvements to health. Sub-saharan Africa, the world's poorest region, still has average life expectancies far below those of the wealthiest countries. Underlying much of this unequal burden of disease is the fact that environmental factors are a major contributor to sickness and death throughout the world, especially in the poorest regions. Human health and sustainable development are inextricably linked. Agenda 21- the United Nations programme of action on sustainable development - comprises a framework for action for sustainable development that focuses on economic, environmental, sociodemographic and health factors ${ }^{1}$.

Development is the process of improving the quality of human life. Such aspects of development as economic development, social development or health development are often considered separately, but all aspects interact and are ultimately part of the same whole. A broad international consensus exists on the general principles that should be followed for development.

Sustainable development encompasses environmental and economic sustenance and sociodemographic and health dimensions. It means development that meets the needs of the present without compromising the ability of future generations to meet their own needs. A complementary definition is improving the quality of life while living within the carrying capacity of supporting ecosystems. The European Commission's "Green paper on the urban environment" states that: Sustainable development is a much broader concept than environmental protection. It implies a concern for future generations and for the longterm health and integrity of the environment. It embraces concern for the quality of life (not just income growth), for equity between people in the present (including prevention of poverty), for inter-generation equity (people in the future deserve an environment which is at least as good as the one we currently enjoy, if not better) and for the social and ethical dimensions of human welfare. It also implies that further development should only take place as long as it is within the carrying capacity of natural systems. Thus, it includes empowerment, equity and health as well as a quality of life that encompasses environment, health and social security. It implies changing existing development strategies, but the details of this are still not clear despite the definition of general principles.

WHO defines health as a state of complete physical, mental and social wellbeing and not merely the absence of disease and infirmity. The enjoyment of the highest attainable level of health is one of the fundamental rights of every human being without distinction of race, religion, political belief, economic or social condition. The quality of the environment and the nature of development are major determinants of health. Health is also an important stimulus to other aspects of development. Healthy people are more productive economically, which increases the economic growth rate of countries; investing in health is thus one means of accelerating development. More importantly, good health is a goal in itself. The WHO Commission on Health and Environment stated that: Human health ultimately depends on society's capacity to manage the interaction between human activities and the 
physical and biological environment in ways that safeguard and promote health but do not threaten the integrity of the natural systems on which the physical and biological environment depends ${ }^{2}$. This includes maintaining a stable climate and the continued availability of environmental resources (soil, fresh water, clean air). Household decisions shape health, but these decisions are constrained by the income and education of the household's members. The dominant risk factors for the most frequent severe diseases are related to various individual characteristics (such as genetic predisposition or other individual susceptibility) and behavioural and lifestyle factors (such as tobacco smoking, psychoactive drugs, nutrition, alcohol consumption and sexual behaviour) ${ }^{3}$. These risk factors combined with a number of environmental factors influence human health. Employment status and satisfaction also affect people's health.

Human beings are at the centre of concerns for sustainable development ${ }^{4}$. They are entitled to a healthy and productive life in harmony with nature. The extent to which sustainable development benefits a community is closely tied to its level of health, as health is a product of economic, social, political and environmental factors, as well as of health services. If our development path is not conducive to sustained improvements in health, then it is not sustainable development. Health, in turn, contributes to economic, social and environmental development through multiple pathways. Improved health feeds sustainable development, and sustainable development feeds improved health in a virtuous cycle, supported by effective health services.

The opposite is experienced by many of the world's people: a vicious cycle of underdevelopment and ill-health. The poor, marginalized, displaced and refugees carry the greatest burden of preventable and treatable disease and death $^{5}$. Inappropriate development and over-consumption also drive the disease burden. Women, children, youth, the elderly, orphans and people with disabilities are amongst those vulnerable to disproportionate burdens of specific forms of ill-health. This holds true at all levels of development, but is most pronounced under circumstances of poverty

Whole communities are often marginalized and excluded from the opportunities for sustainable development and health - be it in rural or urban areas, amongst minority groups, in the face of direct discrimination, or amongst refugees or those displaced by war or conflict. There are health consequences of social exclusion, poorer services and lack of opportunities for development and empowerment. Peace, good governance, political stability and concern for its people are the foundation for the sustainable development of nations ${ }^{6}$.

Over the past decade, there have been improvements in life expectancy and declines in infant and child mortality rates - all key indicators of health. However, these global trends, while highlighting what has been achieved, hide the fact that the gains of development are being reversed in a number of countries, particularly in sub-Saharan Africa ${ }^{7}$. This is strongly associated with the impact of HIV/AIDS, but is also tied to underdevelopment, people becoming poorer, or being negatively affected by war and conflict.

In communicable diseases, notable successes have been achieved against polio, guinea worm (dracunculiasis) and river blindness (onchocerciasis). However conditions such as AIDS, tuberculosis and malaria (which result in approximately 2 million, 1.5 million and 1 million deaths respectively each year), as well as the major communicable diseases of childhood such as acute respiratory infections (predominantly pneumonia), diarrhea and measles (which lead to approximately 4 million, 1.5 million and 800000 deaths respectively each year) ${ }^{7}$. Together they are responsible for more than $90 \%$ of deaths from communicable disease. Malnutrition, including micronutrient deficiency, is associated with more than half of these deaths. The death burden is greatest in subSaharan Africa. Developing countries remain vulnerable to epidemics, such as cholera.

Consequent on unsustainable development, noncommunicable diseases (NCDs) are a significant and growing burden in developing countries. Most noncommunicable disease deaths and high levels of morbidity occur in the developing world. $77 \%$ of deaths from NCDs worldwide occur in developing countries. These include diseases of lifestyle (for example due to unhealthy diets, physical inactivity, tobacco and alcohol use), injuries, violence, mental ill-health, disability and occupation. In developing countries each year, there are around 5.5 million deaths from heart attacks, 5.1 million from strokes and 2.9 million from tobacco-related disease ${ }^{8}$.

Poverty leads to ill-health, but possibly ultimately more debilitating is the negative impact of poor health on development. Malaria alone is estimated to have slowed economic growth in Africa by up to $1.3 \%$ each year and HIV/AIDS by up to $2.6 \%$ in high prevalence countries 9 . These percentages translate into billions of dollars lost. When the consequences of the high burden of other preventable diseases and lack of effective care are added, the result translates into hundreds of billions of dollars. 
Considering what an annual investment of hundreds of billions of dollars would have on life in poorer countries succinctly illustrates how investments in health and health care are productive, and not simply consumptive - as some are prone to think - with more than tangible returns.

Good health enhances development through multiple pathways. This includes survival of trained labour, higher productivity among healthier workers, higher rates of savings and investment, greater enterprise and agrarian productivity and increased direct foreign investment and tourism. Children's educational attainment is higher, which ultimately enhances productivity, lowers rates of fertility and changes the dependency ratio. In short, health is a positive economic asset for countries ${ }^{10}$. Ill-health exacerbates poverty at the family level. The most visible impact is a catastrophic illness or injury which, in the absence of an effective public health service or prepayment system, can lead to a debt trap that impoverishes families for years, driving ill-health in the entire family through mechanisms such as malnutrition. This in turn undermines the potential of families for development.

Poverty is the predominant underlying cause of the huge burden of disease in poorer countries, and the disproportionate burden amongst the poor elsewhere ${ }^{11}$. Poverty resulting in ill-health is multidimensional and requires broad intersectoral interventions. The importance of these intersectoral factors on health is being recognized ever more acutely in international fora and by international organisations, and are now an important feature in poverty reduction strategies. Underdevelopment, unsustainable patterns of development, and production and consumption processes, at both global and local level, are using resources and degrading the environment in a manner that is seriously damaging to health now, and even more so in the future. It has been estimated that poor environmental quality contributes to around $25 \%$ of all preventable illhealth in the world today, the majority of which is poverty related $^{8}$. Rapidly urbanising areas in middle and also lower-income countries face a number of environmentallydriven health problems related to industrialisation, poverty, social dislocation (for example trauma from violence) and lack of utilities. The problems of unplanned human settlements - overcrowded housing, pollution, noise and waste lead to widespread ill-health. In addition, social instability and undermining of moral values are contributing to increased violence, the abuse of women and children, drug and alcohol abuse and mental ill-health. The effects are generally worse in urban fringes and inner cities. Although different hazards and health consequences are frequently associated with levels of poverty, affluence and types of settlement, the relationships vary widely. Also, the future health risks will have an impact beyond the source creating them.

Disease control programmes have the potential to impact massively on the disease burden. Influencing sexual behaviour to prevent HIV/AIDS, treatment compliance for tuberculosis, rapid treatment for malaria, reaching children to immunise them against measles, use of oral rehydration to prevent dehydration from diarrhoea and early identification and treatment of pneumonia are all within our grasp. Programmes and initiatives such as the International Partnership Against Aids in Africa, Stop TB, Roll Back Malaria, the Integrated Management of Childhood Illnesses, and Making Pregnancy Safer are all making a major contribution ${ }^{12}$. However, overall success to date has been limited, because the overall effort has not been of sufficient scale to impact at the level desired. The concept of disease control is commonly erroneously applied only to communicable diseases. The potential for effective prevention and control programmes to impact on non-communicable diseases, such as chronic obstructive airways disease, diabetes, hypertension, myocardial infarction, epilepsy and blindness is similarly massive.

Success in reducing the disease burden requires more than disease control programmes. Besides sustainable economic, environmental and social development, countries also require a solid health care system, capacity for strategic support and effective mobilization of personal action and technological development to improve health. Effective health services are the backbone of health interventions, and have the potential to impact dramatically on health. To be effective, services need to be accessible and offer good quality care. This requires appropriate focus, equitable distribution, good organization and sufficient resources (human, physical and supplies). Yet many countries are unable to secure or sustain their health services at the level required to make the desired impact to effectively support disease reduction ${ }^{13}$. Governance and management weaknesses do continue to compromise the system but, however judiciously available money is spent, current funding levels are inadequate to allow for viable health systems.

The inequity in burden of disease and of development opportunities is mirrored by health services often not being evenly spread between and within countries. As the poorest and most marginalized people and those displaced by war and other emergencies are especially vulnerable and bear a disproportionate burden of disease, if the aim is to massively reduce disease burden, then health care should 
be skewed towards them. Yet, the inverse is generally true. To date, overall efforts to achieve sustainable development and improved health have not been successful enough. A major commitment will be required to allow history to judge this generation as the one that turned the corner on improving the quality of life and health of all the world's people without increasing the use of our natural resources beyond the earth's capacity.

Sustainable development policies should incline us toward longer-term, broad-spectrum interventions, touching upon the driving forces operating in human society. In many developing countries, this would mean tackling inequities, poverty, and population growth and thereby contributing, for example, to the control of land degradation and deforestation, biodiversity loss, soil erosion, food insecurity, and decline in water quality ${ }^{14}$. In developed countries, inequities are also of importance, as sizeable population groups live in squalor and relative poverty. In addition, emphasis should be placed on reducing unsustainable consumption, curbing the use of nonrenewable fuels, and reducing generation of solid wastes to minimize transboundary pollution, toxic waste problems, and global environmental change. All of these actions would have long-term and sustained beneficial effects on human health. To implement successfully proactive preventive approaches, development policies and planning need a long time horizon. In addition, health and environment concerns must become an integral part of the planning within the framework of sustainable development.

\section{References}

1. United Nations. Agenda 21: the United nations programme of action from Rio. New York: United Nations, 1993.

2. World Health Organization. Health and Environment in Sustainable Development: Five Years after the Earth Summit. Geneva: World Health Organization, 1997.

3. Litsios S. Sustainable development is healthy development. World Health Forum 1994;15:193-195.

4. Warford JJ. Environment, health and sustainable development: the role of economic instruments and policies. Bull World Health Organ 1995;73:387- 395.

5. McKeown T. The Role of Medicine: Dream, Mirage or Nemesis? London: Nuffield Provincial Hospitals Trust, 1976.

6. Beaglehole R, Bonita R. Public health at the crossroads: achievements and prospects. Cambridge, United Kingdom: Cambridge University Press, 1997.

7. Brown V, Ritchie J, Rotem A. Health promotion and environmental management: a partnership for the future. Health Promotion Int 1992;7:219- 230. 8. Health for all targets - the health policy for Europe. Copenhagen, 9. WHO Regional Office for Europe, 1991 (European Health for All Series, No. 4). 10. Our planet, our health. Report of the WHO Commission on Health and Environment. Geneva, World Health Organization, 1992.

11. World Commission on Environment and Development. Our common future. Oxford, Oxford University Press, 1987.
12. European Commission Expert Group on the Urban Environment. European sustainable cities. Brussels, European Commission, 1994 (DGXI/822/94-EN). 13. World Conservation Union/United Nations Environment Programme/World Wide Fund For Nature.Caring for the Earth - a strategyfor sustainable living. Gland,Switzerland, World Conservation Union, 1991.

14. Commission of the European Communities. Green paper on the urban environment. Brussels, Office for Official Publications of the European Communities, 1990, p. 59 (CB-CO-90-276-EN-C). 